\title{
Trypanosoma cruzi in dogs: electrocardiographic and echocardiographic evaluation, in Malinalco, State of Mexico
}

This article was published in the following Dove Press journal:

Research and Reports in Tropical Medicine

17 December 201।

Number of times this article has been viewed

\author{
Sandra Díaz González- \\ Vieyra' \\ Ninfa Ramírez-Durán² \\ Ángel H Sandoval-Trujillo ${ }^{3}$ \\ Juan C Vázquez-Chagoyán' \\ Humberto G Monroy- \\ Salazar ${ }^{\prime}$ \\ Alberto Barbabosa-Pliego' \\ 'Research Center of Advanced \\ Studies in Animal Health, Veterinary \\ Husbandry School, ${ }^{2}$ Medical and \\ Ambiental Microbiology, Research \\ Center of Advanced Studies in \\ Health Science, School of Medicine, \\ Autonomous University of the \\ State of Mexico, Toluca, Mexico; \\ ${ }^{3}$ Department of Biological Systems, \\ Metropolitan Autonomous University, \\ Xochimilco, Mexico City, Mexico
}

Correspondence: Alberto Barbabosa-Pliego Centro de Investigación de Estudios Avanzados en Salud Animal, Facultad de Medicina Veterinaria y Zootecnia, Universidad Autónoma del Estado de México Tel +52 7222965555

Fax +52 7222968980

Email abarbabosa@yahoo.com.mx
Abstract: Chagas disease caused by Trypanosoma cruzi is an important public health problem in Latin America. Dogs are considered a risk factor for human Chagas disease, a sentinel for T. cruzi infection in endemic regions and an animal model to study pathological aspects of the disease. The potential use of dogs as indicators of human cardiac pathogenicity of local T. cruzi strains has been studied insufficiently. We studied electrocardiographic (EKG) and echocardiographic (ECG) alteration frequencies observed in an open population of dogs in Malinalco, Mexico, and determined if such frequencies were statistically associated with T. cruzi infection in dogs. Animals $(\mathrm{n}=139)$ were clinically examined and owners were asked to answer a questionnaire about dogs' living conditions. Two commercial serological tests (IHA, ELISA) were conducted to detect anti-T. cruzi serum antibodies. Significant differences between seropositive and seronegative animals in cardiomyopathic frequencies were detected through EKG and ECG $(P<0.05)$. Thirty dogs $(21.58 \%)$ were serologically positive to anti-T. cruzi antibodies (to ELISA and IHA assays), of which nine (30\%) had EKG and/or ECG alterations. From the remaining 104 (78.42\%) seronegative animals, five (4.5\%) had EKG and/or ECG abnormalities. Our data support the hypothesis that most EKG and ECG alterations found in dogs from Malinalco could be associated with $T$. cruzi infection. Considering the dog as a sentinel and as an animal model for Chagas disease in humans, our findings suggest that the T. cruzi strains circulating in Malinalco have the potential to produce cardiomyopathies in infected humans.

Keywords: chagas disease, Trypanosoma cruzi, cardiomyopathy, electrocardiography, echocardiography, Malinalco, México

\section{Introduction}

Chagas disease is caused by Trypanosoma cruzi and is transmitted by a hematophagous insect vector ("kissing bug") of the Reduviidae family. Approximately 10 million people are infected with T. cruzi in 19 countries in Latin America and 50,000,000 people live at risk of infection. ${ }^{1}$ In Mexico, 1.6-5.8 million people may be infected with T. cruzi. $^{2-5}$ Our group has been studying Chagas disease in the State of Mexico, located under the Tropic of Cancer, where most critical transmission areas in Mexico are located. ${ }^{4,5}$ In a previous study we reported T. cruzi prevalences of $7.1 \%$ and $21 \%$ for humans and dogs, respectively, ${ }^{6}$ and more recently we have described an epidemiologic study using dogs and triatomines to assess parasite circulation in the Tejupilco municipality. ${ }^{7}$ Malinalco is a town located in the south-center region of the State of Mexico, from which no previous reports on T. cruzi circulation have been published. However, it is a neighboring area of Tejuplico municipality and shares geographic characteristics with this region. It is also a neighbor of the State of Morelos, where T. cruzi circulation has been 
previously reported, ${ }^{6-10}$ and from Zumpahuacan, from which a pathogenic $T$. cruzi strain has been previously reported and characterized. ${ }^{11}$ In the latter study we used dogs as a model to compare the pathogenicity of a regional T. cruzi strain vs a reference strain (Sylvio X-10), and described clinical (electrocardiographic and echocardiographic) and pathological (macroscopic and microscopic) cardiac alterations caused by these strains and found that, although the two strains were pathogenic, they had differences in virulence, as reported for other strains. ${ }^{12}$ Therefore, epidemiologic studies of Chagas disease in a specific geographical area should consider the pathogenicity of the regional circulating $T$. cruzi strains. Dogs are considered an excellent animal model to study Chagas disease since it mimics the clinical and pathological signs of the disease in humans. ${ }^{13,14}$ Accordingly, the objectives of the present study were: first, to use the house-owned dogs from Malinalco to evaluate the feasibility to use them as sentinels to determine prevalence of T. cruzi infection in humans in the city and, second, to evaluate the pathogenicity of the circulating strains with an electrocardiographical and echocardiographical epidemiologic study.

\section{Animals, materials, and methods Study area}

Malinalco (Figure 1) is located in the south-eastern area of the State of Mexico (between $19^{\circ} 01^{\prime} 58^{\prime \prime}$ to $18^{\circ} 45^{\prime} 18^{\prime \prime} \mathrm{N}$ and $99^{\circ} 35^{\prime} 24^{\prime \prime}$ to $99^{\circ} 25^{\prime} 34^{\prime \prime} \mathrm{W}$ ) with an average altitude of $1750 \mathrm{~m}$. It has seasonal climate variations (dry season November-May and rainy season June-October) with an average annual temperature of $20^{\circ} \mathrm{C}$. According to the 2005
National Census Program, ${ }^{15}$ Malinalco has a population of 22,970 and the main economic activities are agriculture, livestock production, and tourism. According to the 2008 State of Mexico Rabies Vaccination Program, Malinalco has a total population of 2160 house dogs.

\section{Animals}

House-owned dogs $(n=139)$ from Malinalco were studied to assess the prevalence of T. cruzi infection in these animals and to study the impact of the infection on dogs' heart conditions. The sample size was calculated with free software "Sample Size Calculator Software", ${ }^{16}$ with the following parameters: 95\% confidence interval, 5\% error, a universe of 2160 house dogs, and 10\% estimated prevalence of T. cruzi infection. All dogs were evaluated serologically for anti-T. cruzi antibodies and electrocardiographically and echocardiographically for heart health. Dogs were included in the study after personalized owners-informed invitation and consent to participate in the study. Owners were asked to answer a brief questionnaire to gather information regarding dogs' breed, weight, sex, age, health status and habits to access the house, existence of triatomines (kissing bug) within or around the house, and pesticide spraying frequency, if any. Additionally, all dogs had to go through a veterinarian physical examination to evaluate their general health status.

\section{Sample collection}

Dogs were handled in the presence of their owners to keep stress and risk to animals and handlers at a minimum. Blood samples $(7 \mathrm{~mL})$ were obtained by puncture of the cephalic

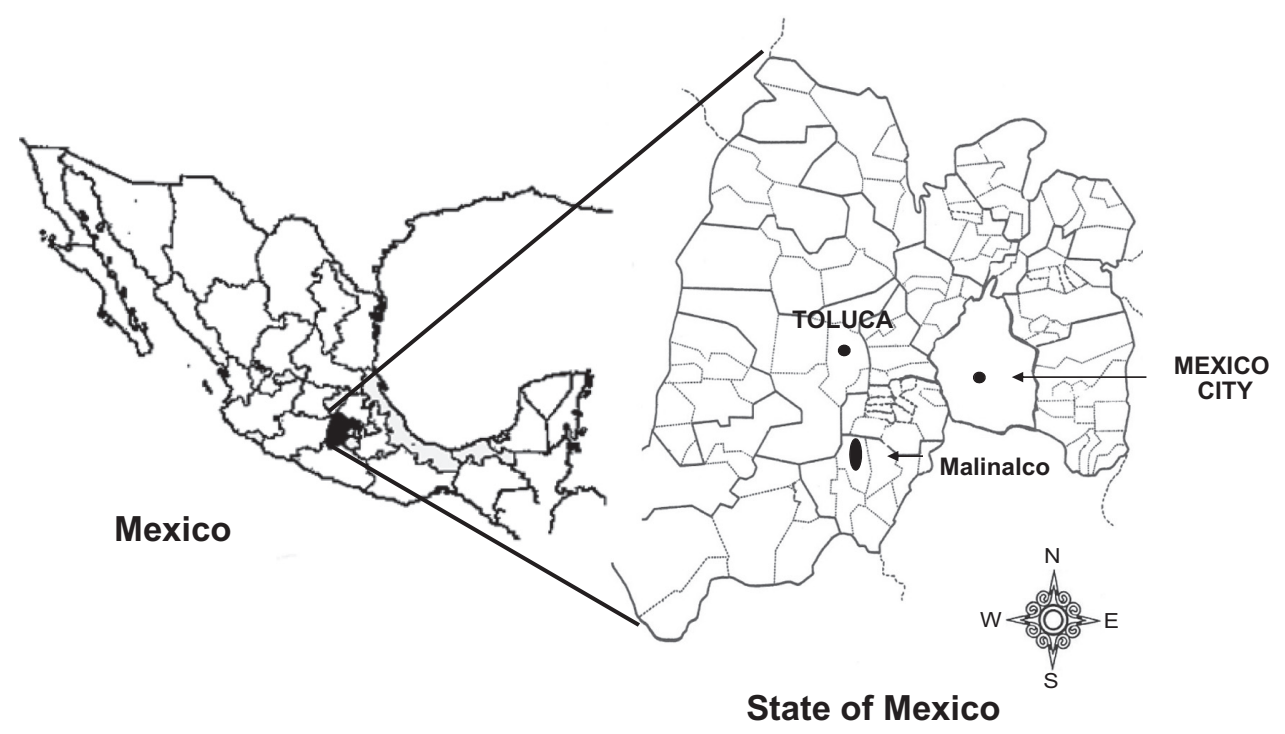

Figure I Location of Malinalco, State of Mexico. 
vein and collected into tubes (Vacutainer ${ }^{\circledR}$, Becton-Dickinson, Mexico, DF, Mexico), sera was obtained by centrifugation at $2000 \mathrm{rpm}$ for 20 minutes, and stored at $-20^{\circ} \mathrm{C}$ until use. Sera samples were analyzed for anti-T. cruzi antibodies by IHA (Polychaco, Laboratorio-Lemos SRL, Buenos Aires, Argentina) (with 98\% sensitivity and 99\% specificity according to the manufacturer's specifications) and ELISA (Laboratorio-Lemos SRL, Buenos Aires, Argentina) Chagas diagnostic kits (with $100 \%$ sensitivity and $100 \%$ specificity, according to the manufacturer's specifications). All assays were performed according to manufacturer's instructions, except that the HRP-labeled, anti-human IgG antibody in the ELISA kit was replaced with HRP-labeled anti-dog IgG (Koma Biotech, Seoul, Korea) as a secondary antibody. ${ }^{11}$ The cut-off value for IHA was set at $\geq 1: 16$ serum dilution and for ELISA at $0.129 \mathrm{OD}_{450 \mathrm{~nm}}+0.1$ (or $+2-\mathrm{SD}$ ). Samples were considered positive when reactive for both assays.

\section{Electrocardiography (EKG)}

Changes in cardiac rhythm and electrical conduction were monitored in all dogs with an EK-8 electrocardiographic machine (Burdick Stylus, Milton, WI), set at 120V, $60 \mathrm{~Hz}$, 20 amps, and 25 watts, and six leads were considered at $25 \mathrm{~mm} /$ second at $1 \mathrm{mV}$, standardized to $1 \mathrm{~cm}$ (Figure 2).

\section{Echocardiography (ECG)}

An ultrasonograph machine (SSD-500, $5 \mathrm{MHz}$ probe; Aloka, Wallingford, CT), with two-dimensional and M-mode functions was used to analyze ventricular function through short-axis images and right parasternal views (Figure 3).

Statistical analysis was conducted through contingency tables and using EPIDAT (v 3.1, 2006, OPS/OMS). Where the immunologic outcome (seropositive or seronegative) was contrasted vs the following variables: EKG (cardiomyopathy or normal), ECG (cardiomyopathy or normal), age $(<1$, $1-2.9,3-6.9$ or $>7$ years old), housing (indoor or outdoor), sex (male or female), and weight ( $<10$ or $>10 \mathrm{~kg})$. Statistical analysis included odd ratios (OR), prevalence ratio (PR) and Chi square $\left(\chi^{2}\right)$, with $95 \%$ confidence intervals and $P<0.05$.

\section{Results}

Dogs' physical examination showed that all animals were apparently healthy. However, the study found $21.58 \%$ (30/139) dogs seropositive to anti-T. cruzi IgG antibodies through IHA and ELISA serology tests.

Differences in the frequency of electrocardiographic and echocardiographic abnormalities were found $(P<0.05)$ between seropositive and seronegative animals, where 9/30 (30\%) and 5/109 (4.5\%) dogs presented electrocardiographic abnormalities (Table 1; Figure 2), and 10/30 (33.3\%) and $17 / 109$ (15.5\%) dogs resulted with echocardiographic abnormalities for seropositive and seronegative animals, respectively (Table 2; Figure 2). The main abnormalities found in seropositive dogs with echocardiography were: right ventricle dilation in $13.3 \%(4 / 30)$, increased motility and or asyn-

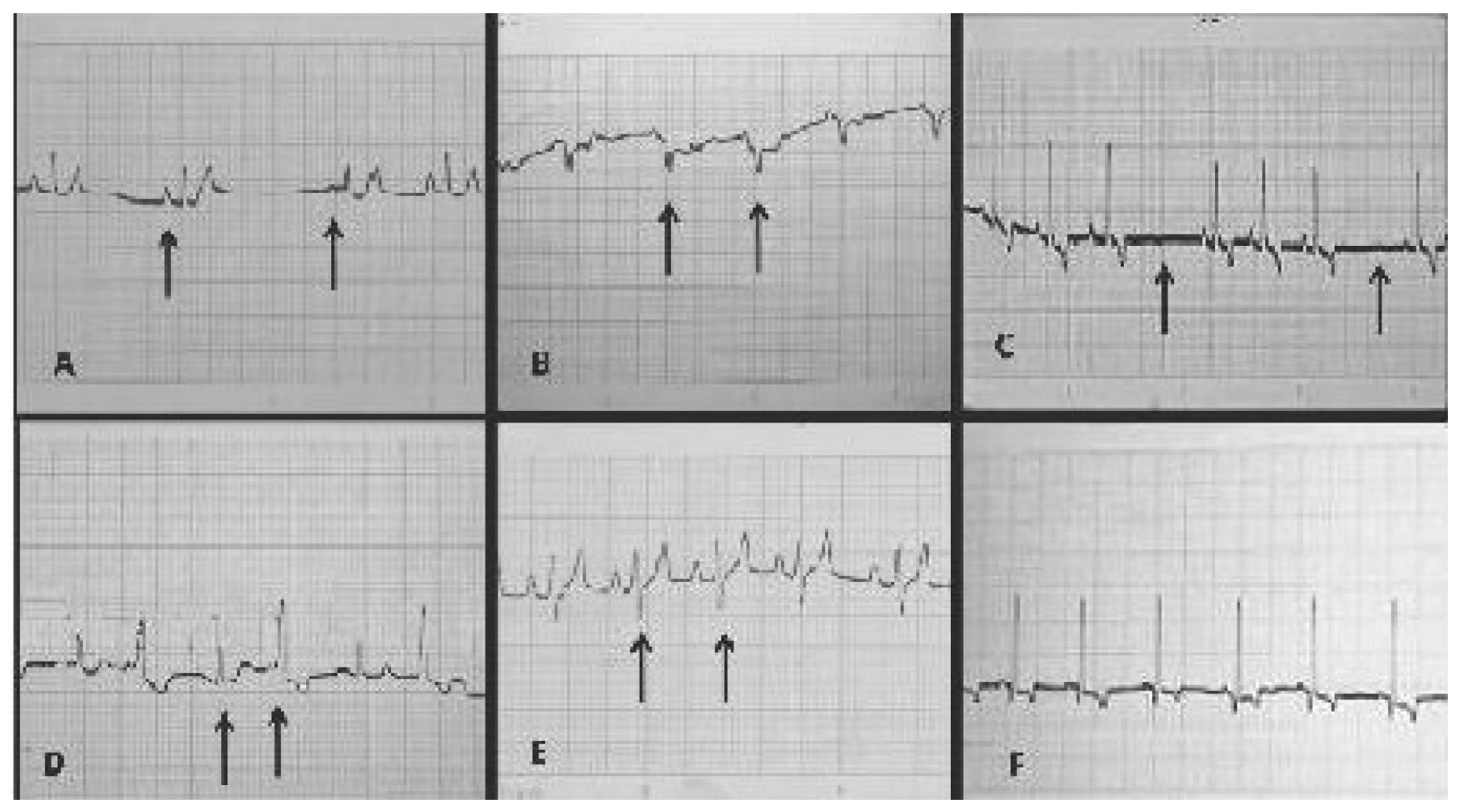

Figure 2 Electrocardiographic analysis in DIl at $25 \mathrm{~mm}$, of seropositive dogs to Trypanosoma cruzi, in Malinalco village, State of Mexico. (A) Sinus pacemaker; (B) aberrant conduction complex QRS; (C) sinus arrhythmia, normal in dogs; (D) wave R, electric alternating; (E) wave S deep; (F) normal. 

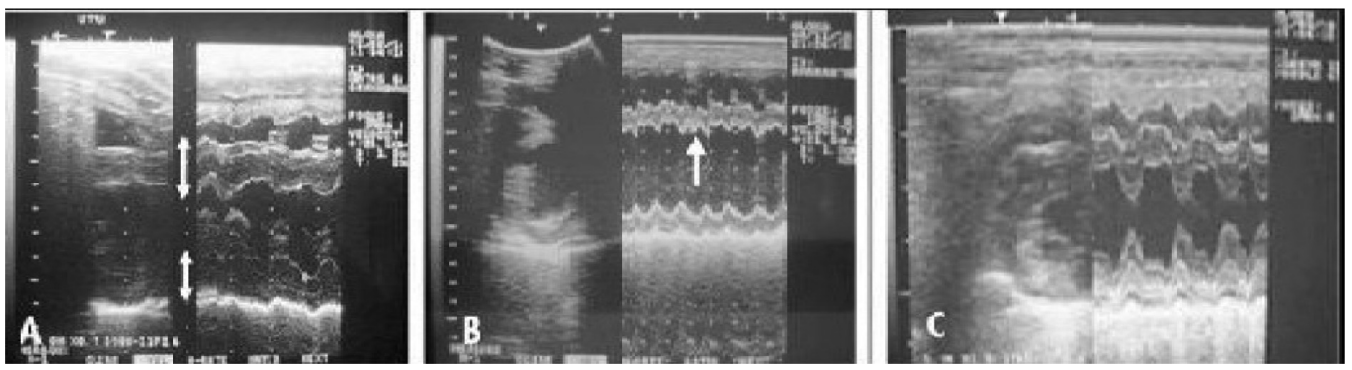

Figure 3 BM-mode echocardiography of seropositive dogs to Trypanosoma cruzi, in the Malinalco village, State of Mexico. (A) Increased thickness of the interventricular septum and left ventricular wall; (B) asynchronic interventricular septum motility; (C) normal.

chrony of the interventricular septum in $13.3 \%(4 / 30)$, left ventricle dilated in $13.3 \%(4 / 30)$, and left ventricular wall thickened in $26.6 \%(8 / 30)$. Echocardiographic abnormalities from seronegative dogs were similar to those found in seropositive dogs but at a lower frequency (Table 2; Figure 3).

Factors such as age, housing conditions, weight, and sex were examined for association with seropositivity (Tables 3-6). No statistical differences could be found for these variables, however some tendencies could be observed in the age of the animals and outdoor housing conditions, which were associated with larger prevalences, suggesting that these variables could be considered as possible risk factors in future studies which should be performed at a larger scale. Overall, a larger proportion of female animals were sampled (86:53 female/male), and a larger proportion

Table I Electrocardiographic alterations found in Trypanosoma cruzi seropositive and seronegative dogs from Malinalco village, State of Mexico

\begin{tabular}{|c|c|c|c|c|}
\hline \multirow[t]{3}{*}{ Electrocardiography } & \multicolumn{4}{|c|}{ Dogs } \\
\hline & \multicolumn{2}{|c|}{$\begin{array}{l}\text { Seropositive } \\
(n=30)\end{array}$} & \multicolumn{2}{|c|}{$\begin{array}{l}\text { Seronegative } \\
(n=109)\end{array}$} \\
\hline & $\mathbf{n}$ & $\%$ & $\mathbf{n}$ & $\%$ \\
\hline Abnormal & 9 & $30^{\mathrm{a}}$ & 5 & $4.5^{b}$ \\
\hline \multirow[t]{2}{*}{ Normal } & 21 & 70 & 104 & 95.5 \\
\hline & 30 & $100 \%$ & 109 & $100 \%$ \\
\hline $\begin{array}{l}\text { Atrium-ventricular } \\
\left.\text { block ( }{ }^{\circ} \text { grade }\right)\end{array}$ & 1 & 3.3 & 0 & 0 \\
\hline $\begin{array}{l}\text { Supra-ventricular arrhythmia } \\
\text { (wandering sinus pacemaker) }\end{array}$ & 3 & 10 & 2 & 1.8 \\
\hline $\begin{array}{l}\text { Ventricular arrhythmia (ectopic } \\
\text { focus/electric alternating) }\end{array}$ & 2 & 6.6 & 1 & 0.9 \\
\hline Right bundle branch block & 3 & 10 & 1 & 0.9 \\
\hline T wave (high) & 0 & 0 & 1 & 0.9 \\
\hline R wave (small) & 8 & 26.6 & 3 & 2.7 \\
\hline
\end{tabular}

Notes: $\chi^{2}$ value $=26.836$, with I $d f ; O R=15.600 ; P R=9.342 . P<0.05 . \chi^{2}$ shows the difference between groups, this difference can be interpreted as an association between seropositivity and risk of developing a cardiomyopathy since OR and PR support analysis risk.
(25.5\%) of this group was seropositive when compared to males (15.0\%). However, no statistical difference was found. The analysis of the influence of dogs' breed on seropositivity prevalence was not possible because the sample size was not large enough due to the diversity of breeds found in the study.

\section{Discussion}

The use of dogs as sentinels to assess human risk of infection was first proposed by Gürtler and colleagues. ${ }^{13}$ More recently, our group demonstrated that $T$. cruzi is found in humans, dogs, and triatomines and found a direct correlation of anti-T. cruzi sero-prevalence between humans (7\%) and dogs (21\%) in the southern region of the State of Mexico. ${ }^{6-8}$ We have also previously demonstrated that regionally circulating $T$. cruzi strains are pathogenic for dogs, either after natural or experimental infections. ${ }^{11}$

Table 2 Echocardiographic alterations found in Trypanosoma cruzi seropositive and seronegative dogs from Malinalco village, State of Mexico

\begin{tabular}{|c|c|c|c|c|}
\hline \multirow[t]{3}{*}{ Echocardiography } & \multicolumn{4}{|c|}{ Dogs } \\
\hline & \multicolumn{2}{|c|}{$\begin{array}{l}\text { Seropositive } \\
(n=30)\end{array}$} & \multicolumn{2}{|c|}{$\begin{array}{l}\text { Seronegative } \\
(n=109)\end{array}$} \\
\hline & $\mathbf{n}$ & $\%$ & $\mathbf{n}$ & $\%$ \\
\hline Abnormal & 10 & 33.3 & 17 & 15.5 \\
\hline \multirow[t]{2}{*}{ Normal } & 20 & 66.7 & 92 & 84.5 \\
\hline & 30 & $100 \%$ & 109 & $100 \%$ \\
\hline RV (size increment) & 4 & 13.3 & & \\
\hline $\begin{array}{l}\text { IS (size increment/ } \\
\text { asynchronic motility) }\end{array}$ & 4 & 13.3 & & \\
\hline IS (size increment) & & & 4 & 3.6 \\
\hline LV (size increment) & 4 & 13.3 & 6 & 5.5 \\
\hline LVW (size increment) & 8 & 26.6 & 14 & 12.8 \\
\hline
\end{tabular}

Notes: $\chi^{2}$ value $=4.728$, with I df; $\mathrm{OR}=2.705 ; \mathrm{PR}=2.137 . \mathrm{P}<0.05 . \chi^{2}$ shows the difference between groups, this difference can be interpreted as an association between seropositivity and risk of developing a cardiomyopathy since OR and PR support analysis risk.

Abbreviations: RV, right ventricle; IS, interventricular septum; LV, left ventricle; LVW, left ventricular wall. 
Table 3 Age associated with seropositivity to T. cruzi in dogs from Malinalco village, State of Mexico

\begin{tabular}{lllllll}
\hline Age (years) & \multicolumn{2}{c}{ Seropositive } & & \multicolumn{2}{c}{ Seronegative } & Total \\
\cline { 2 - 3 } & $\mathbf{n}$ & $\%$ & & $\mathbf{n}$ & $\%$ & \\
\hline$<1$ & 0 & 0.0 & & 21 & 100 & 21 \\
$1.1-3$ & 8 & 16.0 & & 42 & 84.0 & 50 \\
$3.1-7$ & 10 & 28.5 & & 25 & 71.5 & 35 \\
$7.1-10$ & 12 & 36.36 & & 21 & 63.63 & 33 \\
Total & 30 & 21.6 & & 109 & 78.4 & 139 \\
\hline
\end{tabular}

Notes: $\chi^{2}$ value $=11.57 \mathrm{I}$, with I df; PR for level I $=0.0$, PR for level $2=0.166$, PR for level $3=0.285$, PR for level $4=0.363$. $P<0.05$. Even though $\chi^{2}$ shows differences between groups, this difference can only be interpreted as a tendency, since PR does not support analysis risk.

Here we report a $21.58 \%$ seroprevalence anti-T. cruzi in dogs found in Malinalco (a tourist town in a municipality of the southern region of the State of Mexico, with no previous seroprevalence reports). These findings agree with our previous reports in the southern region of the State of Mexico, and considering climatic, geographic, and ecological similarities of the regions studied, this outcome is not surprising. Here we also report the association of seroprevalence of IgG anti-T. cruzi antibodies found in dogs from Malinalco and the occurrence of electrocardiographic and echocardiographic abnormalities in this species. We also analyzed some variables that have been associated with the seroprevalence of dogs to T. cruzi, as risk factors; such as age, weight, sex, and housing.

Through EKG and ECG we found that T. cruzi seropositive animals develop cardiac abnormalities with higher frequencies than seronegative dogs. This finding was expected, since it has been previously demonstrated by us and others that this parasite induces cardiac abnormalities in dogs, and that some T. cruzi strains found in the south of the State of Mexico are cardiopathogenic for dogs. ${ }^{711}$ It is of general knowledge that cardiomyopathies can be found through EKG and ECG among apparently healthy, uninfected, open populations of dogs. The type and frequencies of these pathologies may vary according to the

Table 4 Housing associated to seropositivity to Trypanosoma cruzi in dogs from Malinalco village, State of Mexico

\begin{tabular}{lllllll}
\hline Dog housing & \multicolumn{2}{c}{ Seropositive } & & \multicolumn{2}{c}{ Seronegative } & Total \\
\cline { 2 - 3 } & $\mathbf{n}$ & $\%$ & & $\mathbf{n}$ & $\%$ & \\
\hline Indoors & 2 & 6.5 & & 29 & 93.5 & 31 \\
Outdoors & 28 & 25.92 & & 80 & 74.07 & 108 \\
Total & 30 & 21.6 & & 109 & 78.4 & 139 \\
\hline
\end{tabular}

Notes: $\chi^{2}$ value $=5.39$, with I df; OR $=0.19 ; \mathrm{PR}=0.25 . P<0.05$. Even though $\chi^{2}$ shows the difference between groups, this difference can only be interpreted as a tendency, since OR and PR do not support analysis risk.
Table 5 Body weight associated to seropositivity to Trypanosoma cruzi in dogs from Malinalco village, State of Mexico

\begin{tabular}{|c|c|c|c|c|c|}
\hline \multirow[t]{2}{*}{ Weight (kg) } & \multicolumn{2}{|c|}{ Seropositive } & \multicolumn{2}{|c|}{ Seronegative } & \multirow[t]{2}{*}{ Total (\%) } \\
\hline & $\mathbf{n}$ & $\%$ & $\mathbf{n}$ & $\%$ & \\
\hline$\leq 10.0$ & 2 & 9.5 & 19 & 90.5 & 21 \\
\hline$>10$ & 28 & 24.6 & 90 & 75.4 & 118 \\
\hline Total & 30 & 21.6 & 109 & 78.4 & 139 \\
\hline
\end{tabular}

No difference was found between groups.

breed and age. ${ }^{17,18}$ Here we report electrocardiographic and echocardiographic alterations in an open population of dogs and analyzed their relationship with the seroprevalence of IgG antibodies against $T$. cruzi. The prevalence of cardiac abnormalities among house dogs living in Malinalco was $10.07 \%$. However, as predicted, cardiomyopathic frequencies were different $(P<0.05)$ among anti-T. cruzi seropositive (30\%) and seronegative (4.5\%) animals (Tables 1 and 2). These findings support our previous report in which we concluded that $T$. cruzi strains circulating in the southern region of the State of Mexico are pathogenic to dogs. ${ }^{11}$

Dogs have been considered sentinels for T. cruzi circulation in endemic areas, ${ }^{12}$ and it has also been proposed as a model for Chagas disease since infected dogs mimic some of the signs and pathologies seen in humans, ${ }^{12-14}$ therefore we considered it interesting to evaluate the dog as a sentinel, so that in future it could be used as a tool to estimate human risk of developing cardiomyopathies derived from infection with local T. cruzi strains in different endemic regions. For instance, in a previous study, we reported a correlation of $1: 3$ between human (7.1\%) and dog (21\%) anti-T. cruzi seroprevalences in the southern region of the State of Mexico. ${ }^{6}$ Therefore, assuming that the same correlation exists in cardiac abnormalities due to T. cruzi infection, we could predict that from an estimated population of 2160 house dogs and 22,970 people living in Malinalco, ${ }^{15}$ there should be around 444 and 1608 individuals infected, and

Table 6 Sex associated to seropositivity to Trypanosoma cruzi in dogs from Malinalco village, State of Mexico

\begin{tabular}{lllllll}
\hline Sex & \multicolumn{2}{l}{ Seropositive } & & \multicolumn{2}{c}{ Seronegative } & Total \\
\cline { 2 - 3 } & $\mathbf{n}$ & $\%$ & & $\mathbf{n}$ & $\%$ & \\
\hline Male & 8 & 15.0 & & 45 & 85.0 & 53 \\
Female & 22 & 25.5 & & 64 & 74.5 & 86 \\
Total & 30 & 21.6 & & 109 & 78.4 & 139 \\
\hline
\end{tabular}

Notes: $\chi^{2}$ value $=2.125$, with I df; $\mathrm{OR}=0.517 ; \mathrm{PR}=0.590 . P<0.05$. No difference was found between groups. 
113 and 410, individuals with cardiomyopathies derived from $T$. cruzi infection for dogs and humans, respectively. This estimate is merely speculative and further research is necessary to obtain conclusive evidence, however this approach could be helpful as an indirect method to estimate epidemiologic information necessary for the design of official public health programs.

When we analyzed some variables that could be participating as risk factors of infection, we were able to find some tendencies of association but not statistically signification correlations. Previous studies have reported age as an accumulative risk factor of infection. ${ }^{19-21}$ In this study we observed a strong tendency of age to be associated to seroprevalence, however no statistical significance was found $(P>0.05)$ (Table 3$)$. Future studies should include a larger sample size, to better estimate age as a risk factor. Another factor that has been reported as a risk factor is dog housing (indoor-outdoor). In this study we were not able to demonstrate that housing (indoor vs outdoor), is a risk factor in this endemic region. But, we observed that most owned dogs involved in the present study (77.69\%), are regularly kept outside the house or are able to go in and out at their own will; all of these dogs were considered outdoor animals, and the animals that were kept mostly inside the house were included in the indoor group; the prevalences were $6.5 \%$ and $25.92 \%$ for indoor and outdoor groups, respectively (Table 4). A tendency to have a lower seroprevalence was observed in dogs that were maintained exclusively indoors when compared to outdoor dogs. Most likely a larger sample size is necessary to demonstrate the differences associated with the housing risk factor. On the other hand, other variables analyzed, such as dog weight and sex, do not seem to play important roles in the likelihood of infection (Tables 5 and 6).

We conclude that a relatively high anti-T. cruzi antibody seroprevalence in house-owned dogs from Malinalco village is related to elevated frequencies of electrocardiographic and echocardiographic abnormalities. Further studies should be conducted to fully evaluate dogs as sentinels of infectioninduced, cardiac abnormalities in humans.

Furthermore, our observations emphasize the need of state health agencies to conduct more aggressive, epidemiologic surveillance programs and implement vector control strategies in the State of Mexico, since the seroprevalence found in dogs suggests that there is a latent risk of human infection in Malinalco.

\section{Acknowledgment}

This work was supported by grants of (FE039/2008) SIyEA of Universidad Autónoma del Estado de México.

\section{Disclosure}

The authors report no conflicts of interest in this work.

\section{References}

1. Schofield CJ, Jeannine J, Salvatella R. The future of Chagas disease control. Trends Parasitol. 2006;22:583-588.

2. Velasco-Castrejón O, Guzmán-Bracho C. [Importance of Chagas disease in Mexico]. Rev Latinoam Microbiol. 1986;28:275-283. Spanish.

3. Velazco-Castrejón O. [Chagas disease seroepidemiology in Mexico]. Mexico's Public Health Journal. 1992;34:186-196. Spanish.

4. Guzmán-Bracho C. Epidemiology of Chagas disease in Mexico: an update. Trends Parasitol. 2001;17:372-376.

5. Cruz-Reyes A, Pickering-López JM. Chagas disease in México: an analysis of geographical distribution during the past 76 years - a review. Mem Inst Oswaldo Cruz. 2006;101:345-354.

6. Estrada-Franco JG, Vandanajay B, Díaz-Albiter H, et al. Human Trypanosoma cruzi infection and seropositivity in dogs, México. Emerg Infect Dis. 2006;12:624-630.

7. Barbabosa-Pliego A, Campos P, Olivares D, et al. Prevalence of Trypanosoma cruzi in dogs (Canis familiaris) and triatomines during 2008 in a sanitary region of the State of Mexico, Mexico. Vector Borne Zoonotic Dis. 2011;11:151-156.

8. Medina-Torres I, Vázquez-Chagoyán JC, Rodríguez-Vivas RI, Montes de Oca-Jiménez R. Risk factors associated with triatomines and its infection with Trypanosoma cruzi in rural communities from the southern region of the State of Mexico, Mexico. Am J Trop Med Hyg. 2010;82:49-54.

9. Cortés Jiménez M, Nogueda Torres B, Alejandre Aguilar R, Isita Torneli L, Ramírez Moreno E. Frequency of triatomines infected with Trypanosoma cruzi collected in Cuernavaca city, Morelos, México Rev. Latinoam Microbiol. 1996;38:115-119.

10. Bautista NL, García de la Torre GS, de Haro-Arteaga I, Salazar-Shettino PM. Importance of Triatoma pallidipennis (Hemiptera: Rediviidae) as a vector of Trypanosoma cruzi (Kinetoplasmida: Trypanosomatidae) in the state of Morelos, Mexico, and possible ecotypes. J Med Entomol. 1999;36:233-235.

11. Barbabosa-Pliego A, Díaz-Albiter HM, Ochoa-García L, et al. Trypanosoma cruzi circulating in the southern region of the State of Mexico (Zumpahuacan) are pathogenic: a dog model. Am J Trop Med Hyg. 2009;81:390-395.

12. Andrade ZA. The canine model of Chagas disease. Mem Inst Oswaldo Cruz. 1984;79:77-83.

13. Gürtler RE, Solard ND, Lauricela MA, Haedo M. Dynamic of transmission of Trypanosoma cruzi in a rural area of Argentina. III Persistence of T. cruzi parasitemia among canine reservoirs in a two-years follow-up. Rev Inst Med Trop Sao Paulo. 1986;28: 213-219.

14. Caliari MV, Machado R, Lana M, et al. Quantitative analysis of cardiac lesion in chronic canine Chagasic cardiomiopathy. Rev Inst Med Trop Sao Paulo. 2002;44:273-278.

15. INEGI. 2005. National Institute of Statistic, Geography and Informatics (INEGI). Available at: http://www.inegi.org.mx. Accessed August 11, 2011.

16. Roasft. Roasoft. Sample size calculator. Raosoft, Inc. 2004. Available at: http://www.raosoft.com/samplesize.html. Accessed January 28, 2008.

17. Dukes-McEwan J, Borgarelli M, Tidholm A, Vollmar AC, Häggström J. Proposed guidelines for the diagnosis of canine idiopathic dilated cardiomyopathy. J Vet Cardiol. 2003;5:7-19.

18. Oyama MA, Sisson DD, Solter PF. Prospective screening for occult cardiomyopathy in dogs by measurement of plasma atrial natriuretic peptide, B-type natriuretic peptide, and cardiac troponin-I concentrations. Am J Vet Res. 2007;68:42-47. 
19. Gürtler RE, Cecere MC, Lauricella MA, et al. Incidence of Trypanosoma cruzi infection among children following domestic reinfestation after insecticide spraying in rural northwestern Argentina. Am J Trop Med Hyg. 2005;73:95-103.

20. Diosque P, Padilla AM, Cimino RO, et al. Chagas disease in rural areas of Chaco Province, Argentina: epidemiologic survey in humans, reservoirs, and vectors. Am J Trop Med Hyg. 2004;71:590-593.
21. Pascon da EJP, Pereira NG, Sousa GM, Junior DP, Camacho AA. Clinical characterization of chagasic cardiomyopathy in dogs. Pesq Vet Bras. 2010;30:115-120.

\section{Publish your work in this journal}

Research and Reports in Tropical Medicine is an international, peerreviewed, open access journal publishing original research, case reports, editorials, reviews and commentaries on all areas of tropical medicine, including: Diseases and medicine in tropical regions; Entomology; Epidemiology; Health economics issues; Infectious disease; Laboratory science and new technology in tropical medicine; Parasitology; Public health medicine/health care policy in tropical regions; and Microbiology. The manuscript management system is completely online and includes a very quick and fair peer-review system. Visit http://www.dovepress. com/testimonials.php to read real quotes from published authors.

Submit your manuscript here: http://www.dovepress.com/research-and-reports-in-tropical-medicine-journal 